Journal of Magnetism and Magnetic Materials 322 (2010) 2762-2768

Nanopatterning of perpendicular magnetic recording media by low-energy implantation of chemically reactive ions

M.S. Martín-González ${ }^{1}$, F. Briones, J.M. García-Martín

Instituto de Microelectrónica de Madrid, IMM (CNM-CSIC) C/Isaac Newton, 8 (PTM) 28760 - Tres Cantos. Madrid (SPAIN).

\title{
J. Montserrat
}

Institut de Microelectrònica Barcelona, Campus Universitat Autònoma Barcelona, E08193, Bellaterra, (SPAIN)

L. Vila, G. Faini

Laboratoire de Photonique et de Nanostructures LPN-CNRS, Route de Nozay, 91460 Marcoussis, France

A. M. Testa, D. Fiorani,

Istituto di Struttura della Materia, Area della Ricerca di Roma-CNR, C.P. 10, 00016, Monterotondo Scalo, Roma, Italy

H. Rohrmann

Oerlikon Systems, Iramali 18, FL-9496 Balzers, Liechtenstein E-mail: marisol@imm.cnm.csic.es

\begin{abstract}
Magnetic nanopatterning of perpendicular hard disc media with perpendicular anisotropy, but preserving disc surface planarity, is presented here. Reactive ion implantation is used to locally modify the chemical composition (hence the magnetization and magnetic anisotropy) of the $\mathrm{Co} / \mathrm{Pd}$ multilayer in irradiated areas. The

\footnotetext{
${ }^{1}$ Corresponding author
} 
procedure involves low energy, chemically reactive ion irradiation through a resist mask. Among N, P and As ions, $\mathrm{P}$ are shown to be most adequate to obtain optimum bit density and topography flatness for industrial $\mathrm{Co} / \mathrm{Pd}$ multilayer media. The effect of this ion contributes to isolate perpendicular bits by destroying both anisotropy and magnetic exchange in the irradiated areas. Low ion fluences are effective due to the stabilization of atomic displacements levels by the chemical effect of covalent impurities.

Key words: hard drive, perpendicular magnetic recording, CoPd multilayers, multilayer media, nanoimprint lithography.

\section{I.- Introduction}

Data storage is dominated by disc based magnetic recording technology that combines high capacity, reasonable read/write speeds and cost-effectiveness. The storage density has increased spectacularly through the last years as the ability to reduce magnetic bit volumes has improved. However, as the volume is reduced the magnetic anisotropy energy becomes compatible with the thermal energy $\mathrm{k}_{\mathrm{B}} \mathrm{T}$ (superparamagnetic limit) and the magnetic information of the bits gets lost. New technologies and strategies are needed to postpone or circumvent this limit, ${ }^{1}$ such as antiferromagnetically coupled media ${ }^{2,3}$, perpendicular media ${ }^{4}$ (even deposited on topographically prepatterned substrates ${ }^{5}$ ), patterned media $^{6}$, high anisotropy chemically synthesized nanoparticles media ${ }^{7,8}$,to name a few. A review on fabrication challenges for patterned recording media has been published recently. ${ }^{9}$

Patterned perpendicular magnetic media is a combination of methods being investigated to further extend areal bit densities. ${ }^{4,10,11}$ In this approach, the data are recorded and stored in an array of single-domain magnetic bits ("nanomagnets") that 
have been patterned in the magnetic/recording layer. ${ }^{12}$ Extensive studies on patterned magnetic media have been performed primarily on the fabrication methods supporting bit densities of up to $200 \mathrm{Gbit} / \mathrm{in}^{2}{ }^{13}$

In order to pattern the magnetic media different approaches have already been used, like for example: a) Interference lithography (followed by the deposition in resist holes by, for example, sputtering); ${ }^{14}$ b) imprint technology (where a Co/Pt multilayers have been deposited onto a substrate consisting of a pattern of pillars $28 \mathrm{~nm}$ high has been obtained); ${ }^{15} \mathrm{c}$ ) pre-etched wafers (where for example: Si dot with a height of $36 \mathrm{~nm}$ and a width of $100 \mathrm{~nm}$ covered with $\mathrm{Co} / \mathrm{Pt}$ multilayer has been obtained); ${ }^{16}$ d) selfassembling techniques of nanostructures (such as block copolymer where patterned CoCrPt dots of $40 \mathrm{~nm}$ have been obtained ${ }^{17}$ or self-assembled FePt nanoparticles); ${ }^{18}$ e) nano-pattering with ions (the desired pattern is directly written or deposited in the magnetic layer), ${ }^{19}$ f) ion projections direct structuring -IPDS- (for example, using a dose of $2 \times 10^{15} \mathrm{He}^{+} / \mathrm{cm}^{2}$ at $45 \mathrm{keV}$ in $\mathrm{Co} / \mathrm{Pt}$ multilayer $\left.){ }^{20} \mathrm{~g}\right)$ focused ion beam $(30 \mathrm{keV}$ $\mathrm{Ga}^{+}$at a beam current of $1 \mathrm{pA}$ has also been applied either by removing material or by $\mathrm{Ga}^{+}$poisoning); ${ }^{13,21,22} \mathrm{~h}$ ) hard mask (with Ar ion milling to transfer island patterns into underlying magnetic layers); ${ }^{23}$ or, i) ion irradiation through a mask. ${ }^{24}$

The use of ion irradiation through a mask to modify the magnetic properties of films is also of current interest. In particular, this last case has been studied in detail by Chappert et al. ${ }^{25}$; Ferre et al. ${ }^{26}$ (using fluences between $2 \times 10^{14}$ and $2 \times 10^{16}$ ions $/ \mathrm{cm}^{2}$ with $30 \mathrm{keV} \mathrm{He}{ }^{+}$ions); Weller et al. ${ }^{27}$ (employing $700 \mathrm{keV} \mathrm{N}^{+}$ions); Rettner et al. irradiating $\mathrm{Co} / \mathrm{Pt}$ multilayers with different energies and ion species $\left(20 \mathrm{keV} \mathrm{He}{ }^{+}, 2 \mathrm{MeV} \mathrm{He}^{+}\right.$, $20 \mathrm{keV} \mathrm{Ar}{ }^{+}, 2 \mathrm{MeV} \mathrm{Ar}{ }^{+}, 30 \mathrm{keV} \mathrm{Ga}{ }^{+}$using doses $\left.10^{11}-10^{17} \mathrm{ions} / \mathrm{cm}^{2}\right) ;{ }^{28}$ Menendez et al. using broad beam $\mathrm{Xe}^{+}$ion irradiation through lithographically defined mask in $\mathrm{Fe}_{60} \mathrm{Al}_{40}$ alloys. $^{21}$ 
Our nano-patterning approach is also based on ion irradiation through a mask. However, the novelty is to use low ion implantation energies of chemically reactive ions like $\mathrm{P}$ and As (instead of conventional noble gases or nitrogen). The idea is to force the formation of covalent Co-P and Co-As bonds, respectively. The anisotropy in $\mathrm{Co} / \mathrm{Pd}$ multilayers results from d-shell electron hybridization at the interfaces between the cobalt and palladium layers. The formation of covalent bonds with Co will a priori deplete valence electrons from it. Those electrons are not longer available for $\mathrm{d}$-shell electron hybridization with $\mathrm{Pd}$, therefore reducing $\mathrm{Co} / \mathrm{Pd}$ anisotropy locally without modifying topography. In other words, we should be able to preserve surface planarity, but induce magnetic patterning, while maintaining the high throughput of vacuum industrial processing (continuous or modular process).

\section{II: EXPERIMENTAL METHODS}

Multilayered hard disc media for perpendicular recording have been prepared in a commercial sputtering deposition system (Circulus M12) equipped with Triatron cathodes from UNAXIS. "HOYA N5" hard disk substrates of $65 \mathrm{~mm}$ diameter were used as a substrate for the the multilayers of this study. The base pressure prior to deposition was $9 \cdot 10^{-8}$ mbar. $20 \mathrm{~nm} \mathrm{Ti}+10 \mathrm{x}(30 \mathrm{~nm} \mathrm{NiFe}+2 \mathrm{~nm}$ Carbon), which behaves as soft magnetic underlayer -SUL- / $12 \mathrm{~nm}$ Pd (buffer layer)/15x (0.28 nm $\mathrm{Co} / 0.93 \mathrm{~nm} \mathrm{Pd}$ ) (magnetic layer with perpendicular anisotropy)/5 nm diamond carbon (capping layer)) were deposited. A decisive parameter for low contamination of the layers were the high deposition rates (Co: $1 \mathrm{~nm} / \mathrm{sec}, \mathrm{Pd}: 7.2 \mathrm{~nm} / \mathrm{sec})$ and the fast switching between sub-layers $(<2 \mathrm{msec})$. This results in $\sim 6.3 \mathrm{sec}$ for the stack of 15 $\mathrm{Co} / \mathrm{Pd}$ bilayers and the base pressure causes less than half a monolayer contamination in the $18 \mathrm{~nm}$ thick $\mathrm{Co} / \mathrm{Pd}$ layer stack. 
The implantation was performed in an Eaton NV4206 ion implanter, with fluences ranging from $10^{14}$ to $2 \cdot 10^{16}$ ions $/ \mathrm{cm}^{2}$ and energies set at $20-30 \mathrm{KeV}$ for various ions $\left({ }^{14} \mathrm{~N}^{+},{ }^{31} \mathrm{P}^{+}\right.$and $\left.{ }^{75} \mathrm{As}^{+}\right)$. These ions were selected since they could be divided into two types: chemically inert with Co or Pd $(\mathrm{N})$ and chemically active like (P, As), since they can form a covalent bond with cobalt from the alloy.

The ion stopping masks were defined by e-beam lithography by use of a Leica EBPG 5000+ working at $100 \mathrm{keV}$. For this purpose, a negative resist (MAN 2403) was spun onto the samples, with an optimum thickness sufficient to stop each type of ion, so the underlying magnetic layer is protected during the irradiation process except in those areas where the resist was removed. Prior to this step some gold marks were deposited on the sample to define arrays in order to locate the nanostructured areas for measurements purposes. After implantation, the resist mask was removed by oxygen plasma cleaner.

Structural modifications were studied by X-ray diffraction (XRD) using a PHILIPS XPERT four-circle diffractometer. Atomic Force Microscopy (AFM) measured the surface topography. X-ray Reflectrometry (XRR) studied modifications of layer thickness. Wide X-Ray Photoemission Spectroscopy (XPS) were used to determine the film compositions, and narrow XPS scans to analyze the chemical bonding between atoms. AT\% composition was determined using the standard Scofield sensitivity factors. The samples were cleaned using an Ar beam to remove surface contaminants. These analysis were performed using an VG Escalab 250 iXL ESCA instrument (VG Scientific), equipped with aluminum Ka1,2 monochromatized radiation at $1486.92 \mathrm{eV}$ X-ray source. The XPS measurements were carried out using monochromatic Al-K $\alpha$ radiation $(\mathrm{h} v=1486.92 \mathrm{eV})$. Photoelectrons were collected from a takeoff angle of $90^{\circ}$ 
relative to the sample surface. The measurement was done in a Constant Analyzer Energy mode (CAE) with a $100 \mathrm{eV}$ pass energy for survey spectra and $20 \mathrm{eV}$ pass energy for high resolution spectra. Charge referencing was done by setting the lower binding energy $\mathrm{C}$ 1s photo-peak at $285.0 \mathrm{eV} \mathrm{C} 1 \mathrm{~s}$ hydrocarbon peak. The spectra fitting is based on "Chi-squared" algorithm used to determine the goodness of a peak fit. Chisquared $<2$ implies a good fit. Magnetic Force Microscopy (MFM) and commercial SQUID magnetometer were use to analyzed magnetic properties.

\section{III- Results and Discusión}

\section{IIIa: Continuous films}

To understand the effect of implantation with reactive ions on the $\mathrm{Co} / \mathrm{Pd}$ multilayers magnetic properties, continuous films were implanted (without nanopatterning) with N, P, and As. Stopping and Range of Ions in Matter (SRIM) simulations $^{29}$ were used to calculate the appropriate ion energy ranges to cause implantation at the precise depth $(\sim 17.5 \mathrm{~nm}$, according to XRR). As a result, the energy and flux density ranges selected for the experiments were $20-30 \mathrm{keV}$ and $10^{14}-2 \cdot 10^{16}$ ions $/ \mathrm{cm}^{2}$. It is interesting to note here that the multilayers are 15 layers of Co $0.28 \mathrm{~nm}$ thick each. Taking into account the lattice parameter for a cubic cobalt lattice is 0.3545 $\mathrm{nm}^{30}$, one can calculate a concentration of $8 \times 10^{14}$ atoms $/ \mathrm{cm}^{2}$ per monolayer and a total amount of $1.210^{16} \mathrm{Co}$ atoms $/ \mathrm{cm}^{2}$ in the sample. So the fluences employed in this study are in the range of Co concentration. Figure 1 shows structural and magnetic changes after implantation. As checked by XRR, no significant variation was detected on the total thickness of the samples after implantation, except for fluences above $10^{16}$ ions $/ \mathrm{cm}^{2}$ for $\mathrm{N}$ and $\mathrm{P}$, and above $10^{16}$ ions $/ \mathrm{cm}^{2}$ for As, see table I. 
By XRD scans (Figure 1(a)) only one broad peak can be detected at $2 \theta=$ $40.6824^{\circ}$ instead of two (the Co and Pd one). This peak can be interpreted as the (111) of the CoPd alloy ${ }^{31}$ and its intensity can be related to a small interdiffusion between the layers during the growth process. In our case, the intensity of the (111) peak is small and broad which indicates formation of a few grains with that orientation and with a size of 10-15 $\mathrm{nm}$ according to transmission electron microscopy. ${ }^{32}$ After implantation the peak moves to lower $2 \theta$ values $40.1287^{\circ}$ for $2 \cdot 10^{16}$ ions $/ \mathrm{cm}^{2}$, which means that the lattice is expanded due to the implanted atoms. A similar displacement is observed after implantation with $\mathrm{P}$ or As. From AFM experiments, it can be seen that the roughness of the films increases from $0.6 \mathrm{~nm}$ for the as grown samples up to $1.4 \mathrm{~nm}$ for the samples implanted at $30 \mathrm{keV}$ with $10^{15} \mathrm{ions} / \mathrm{cm}^{2}$, this result is better (less roughness) than the obtained by some of the methods presented in the introduction section (like in references 12, 14-16, 21 and 22 to cite some), see figure 2.

Concerning the magnetic properties, the as-deposited samples exhibit square hysteresis loops with high coercive field (above 5000 Oe) when the field is applied perpendicularly to the surface, which is indicative of a perpendicular magnetic anisotropy that can be ascribed to Co and Pd ultrathin layers interface anisotropy. MFM experiments in the pristine samples in the demagnetized state show the maze domain pattern characteristic of perpendicular anisotropy multilayers (see figure 1b). Black and white regions correspond to magnetic domains with perpendicular magnetization pointing upwards and downwards, respectively. With implantation, the main effect is the reduction of the magnetization and the coercive field as shown on Table I. For comparison purposes some of the experimental data from Table I are also shown on figure $1 b$. 
In the case of nitrogen implantation, the material becomes magnetically softer as the dose is increased, see table I. The intermixing of the Co/Pd interfaces (as detected by Rutherford Backscattering) and a possible effect of the C-coating inside the multilayer explain this softening. It is well established that the less abrupt the interfaces, the lower the interface anisotropy. ${ }^{33,34}$ It should be noticed that the squareness of the hysteresis loop is maintained up to $10^{15}$ ions $\mathrm{cm}^{-2}$. The easy axis is still perpendicular to the multilayer plane until $10^{15}$ ions $/ \mathrm{cm}^{2}$ flux densities. ${ }^{32}$ For flux densities above $10^{16}$ ions $\cdot \mathrm{cm}^{-2}$, the saturation magnetization is strongly reduced. The highest concentration of ions implanted at $2 \times 10^{16}$ ions $\mathrm{cm}^{-2}$ is $3.3_{\mathrm{at}} \%$ as determined by $\mathrm{X}$-ray photoemission spectroscopy (XPS), while for lower flux densities no ions were detected by this technique. These measurements are performed in all of the samples after Ar etching to eliminate surface contamination.

Nitrogen atoms, although forming stable compounds with other transition metals like $\mathrm{Fe}^{35-37}$ or $\mathrm{Cu}^{38}$, do not form stable compounds with Co or Pd at room temperature, as detected by XPS, so the effect caused is similar to implant the sample with noble gases like $\mathrm{He}^{+}$or $\mathrm{Ar}^{+}$, like it was done before for $\mathrm{Co} / \mathrm{Pt}$ multilayers ${ }^{39}$ The implantation with chemically reactive ions like $\mathrm{P}$ and As (which forms stable compounds with $\mathrm{Co}^{40}$ ) will represent a major advance. Its implantation is more efficient to lower both anisotropy (by $\mathrm{Co} / \mathrm{Pd}$ interface interdiffusion, as before) and $3 \mathrm{~d}-4 \mathrm{~d}$ hybridization reduction $^{41}$ (exchange reduction). The chemical bonding Co-P or Co-As reduces previously available $d$ sublevels of Co, so the magnetic moment induced from Co to $\mathrm{Pd}$ (due to the $3 d-4 d$ hybridization) is strongly reduced. This behavior explains why $\mathrm{M}_{\mathrm{s}}$ is more efficiently destroyed by lower implantation doses while comparing P and As with $\mathrm{N}$, as shown on Table I. 
Furthermore, since $\mathrm{P}$ is lighter than As, the physical sputtering effect during implantation is minimized, as shown on Table I. So, for the same fluence, the film thickness is better preserved for P than for As. Therefore, phosphorous appears to be the most adequate implantation source for this type of multilayers, from the three ions.

\section{IIIb- Nanostructured samples: Magnetic Nano-patterning without patterning the surface}

The procedure is described in figure 3(a). Firstly, resist was spun onto the sample. Afterwards, the pattern in a $200 \times 200 \mu \mathrm{m}^{2}$ area was prepared; see figure $3(\mathrm{~b})$, and ion implantation with $\mathrm{N}, \mathrm{P}$ and As at the same fluences and doses as the one shown on table I for the un-patterned samples were performed. After implantation, the resist mask is removed in oxygen plasma.

Although for this lab scale experiments the e-beam lithography approach was used, for industrial production a nanoimprint method would be more realistic. Since this technology presents high throughput capability, compatible with in mass production, there is no need for expensive optics. Although the master mould to generate the nanoimprint can be costly nowadays, ${ }^{42}$ the inversion it is not too high since matermaster replication can be made. The aspect ratio of the photoresist columns was optimized for each ion using the SRIM code, see an example in figure $4 \mathrm{~b}$, considering that they should not be too high to avoid folding down of the resist columns, but enough to stop the different ions. Heavier ions like $\mathrm{P}$ or As are again a better choice since the thickness of the polymer mask can be strongly reduced from $200 \mathrm{~nm}$ for $\mathrm{N}$ to $110 \mathrm{~nm}$ for P to $75 \mathrm{~nm}$ for As, according with the SRIM simulations. As an example of the simulation study performed, the case of $\mathrm{P}$ implantation at $20 \mathrm{KeV}$ is shown in figure 4. It can be observed that this energy is enough to obtain a Gaussian distribution of $\mathrm{P}$ ion 
in the $\mathrm{Co} / \mathrm{Pd}$ multilayers region, and that $110 \mathrm{~nm}$ of photoresist is enough to stops those ions, see figure $4 b$.

Figure 5 show representative images of the different steps involved in the magnetic patterning process explained above, in particular for a mask with $150 \times 150 \mathrm{~nm}^{2}$ dots and $210 \mathrm{~nm}$ periodicity and $\mathrm{N}$ implantation at $30 \mathrm{KeV}$ and $10^{15}$ ions $/ \mathrm{cm}^{2}$ fluence. The uncovered areas (matrix) became magnetically soft (please notice that a continuous film implanted with similar parameters exhibited $\mathrm{M}_{\mathrm{s}} \sim 250 \mathrm{emu} / \mathrm{cc}$ and $\mathrm{H}_{\mathrm{c}} \sim 300$ Oe, see table I, although in nanopatterned areas the actual values have not been determined yet), whereas the protected areas (bits) should remain magnetically hard.As a result, after photoresist removal no changes in topography were observed by AFM, Fig. 5c and AFM profile once the resist was removed in Fig. 5e, (in other words, the surface keeps its flatness), whereas a clear effect on the magnetic image can be observed (Fig. $5 \mathrm{~d}$ and magnetic profile in Fig. 5e). As this image corresponds to a remanent magnetic state after saturation, all the bits exhibit the same contrast (i.e. the magnetization is pointing in the same perpendicular direction in all of them), and the magnetic flux is closed through the low anisotropy matrix. This demonstrates that the bits can maintain their magnetization, indicating that the dipolar interaction among bits is not important, since high dipolar interaction would produce a check-board pattern with bits with alternating up and down magnetization. By performing MFM experiments several weeks after the sample was saturated we have checked that the bits still exhibit the same contrast, i.e. they are stable.

It must be noticed that the sample is magnetically nanopatterned with well defined bits but virtually free of topography. This is an advantage for flying heads at low (sub-10 nm) fly heights, since the head can easily detect the bits flying over a flat surface. However, the patterning transfer is not perfect: a reduction of the bit size with respect to 
the initial resist pattern size is observed due to the lateral straggling of the implanted ions. Simulations reveal a lateral straggling of about $25 \mathrm{~nm}$ for $30 \mathrm{keV}$ nitrogen ions. For $\mathrm{P}$ and As the lateral straggling is smaller.

The results shown in Figure 5 correspond to a density of $15 \mathrm{Gbit} / \mathrm{inch}^{2}$. It is important to point out that in optimum conditions (i.e. with better e-beam lithography, resist, implantation parameters and straggling) lower diameter dots could be possible. Possibly, by optimizing the ideas exposed in this work, for example: a) using other covalent implantation ions with high $\mathrm{Z}$ to reduce at maximum the lateral straggling and with the capability of bonding more than one Co. And, b) using lithographic techniques that can achieve higher bit densities $\left(\sim 1 \mathrm{Tbit} / \mathrm{inch}^{2}\right)$. For example: $10 \mathrm{~nm}$ bits with 40 nm spacing (400 Gbit/inch ${ }^{2}$ ) has been shown using nanoimprint technology ${ }^{43}$ and more recently, the possibility of forming highly packed dot-array patterns with a pitch of 20 $\mathrm{nm} \times 20 \mathrm{~nm}$ and a resist thickness of about $13 \mathrm{~nm}$, which corresponds to about 1.6 Tbits/inch ${ }^{2}$ has been demonstrated. ${ }^{44}$

On figure 6, some examples of samples that have been magnetically patterned with different ions are shown, Figure 6(a) for As and Figure 6(b) and (c) for P. Before the MFM experiments, the samples were demagnetized. As a consequence, in contrast to figure 5(d), -nitrogen implanted sample- where bits exhibiting the same contrast one can distinguish both black and white bits, corresponding to up and down magnetization respectively. The fact that a random demagnetized state is obtained for both As and $\mathrm{P}$ implanted samples, instead of a check-board pattern (that is the demagnetized state with lowest energy for an ordered array of interacting bits) indicates that the dipolar interactions between adjacent bit islands are not relevant. An important effect was observed during the MFM experiments in the $\mathrm{P}$ implanted sample. Comparing consecutive scans, we realized that the bits were stable, but the matrix (i.e. the exposed 
regions during the implantation) was modified towards more attractive contrast (darker in the image), as can be clearly seen comparing Figure 6(b) and Figure 6(c). We attribute this effect to tip-induced modifications in the matrix. Since the matrix is a region with low magnetic anisotropy and low coercivity then its magnetization can be modified by the tip stray field, becoming more parallel to the tip magnetization and as a consequence producing more attraction. In fact, the tip stray field is typically higher than 150 Oe. $^{45,46}$ and the coercivity of the matrix is probably below 120 Oe (such value is taken from Table I and corresponds to an implanted continuous film; in the nanostructured sample the coercivity of the implanted areas can be significantly smaller).

We want to emphasize that the presence of these very low anisotropy and low magnetization areas between two neighbouring magnetic bits is important since it is necessary for the exchange coupling to be weak or virtually nil for stable bit writing. ${ }^{47}$ It helps to cut off the exchange force between the bits, leading a "transition region" 48 and therefore diminishing the transition noise. ${ }^{49}$ This is crucial to achieve a high signal-tonoise ratio.

\section{Conclusions}

Low energy chemically reactive ion implantation modifies the structural and the magnetic properties of magnetic multilayers with perpendicular anisotropy (e.g. $\mathrm{Co} / \mathrm{Pd}$ multilayers). For $\mathrm{N}$ ions, interfacial disorder is generated, giving rise to a strong reduction of the local magnetic anisotropy and exchange coupling. A stronger reduction is observed for $\mathrm{P}$ and As ions when a chemical bonding with Co is established. By using a proper masks and flux densities, magnetic recording media with perpendicular anisotropy and magnetically hard bits in a non-magnetic matrix can be fabricated. 
Heavier ions like $\mathrm{P}$ are a better choice in order to optimize damage depth, to reduce polymer mask thickness and to limit lateral ion straggling. In principle, this technique could be a feasible industrial production method for nano-patterning hard disc magnetic media with perpendicular magnetization while preserving surface flatness.

\section{Acknowledgements}

The work was supported by the EC Growth project HIDEMAR (Contract\# G5RD-CT-2002-00731). The authors thank the LPN technological platform for technical support.

\section{Figures and Tables}

Table I. Effect of ion implantation on the thickness and magnetic properties of CoPd multilayers.

\begin{tabular}{|c|c|c|c|c|c|}
\hline $\begin{array}{c}\text { Implanted } \\
\text { ion }\end{array}$ & $\begin{array}{c}\text { Fluency } \\
(\text { ions/cm }\end{array}$ & $\begin{array}{c}\text { Energy } \\
(\mathrm{keV})\end{array}$ & $\begin{array}{c}\text { Final Thickness } \\
(\text { CoPd layer }) \text { in } \AA\end{array}$ & $\begin{array}{c}\mathrm{M}_{\mathrm{s}} \\
(\mathrm{emu} / \mathrm{cc})\end{array}$ & $\begin{array}{c}\mathrm{H}_{\mathrm{c}} \\
(\mathrm{Oe})\end{array}$ \\
\hline As-deposited & 0 & 0 & 160 & 360 & 5430 \\
\hline $\mathrm{N}$ & $10^{14}$ & 30 & 154 & 313 & 1615 \\
\hline $\mathrm{N}$ & $10^{15}$ & 30 & 153 & 255 & 307 \\
\hline $\mathrm{N}$ & $2 \cdot 10^{16}$ & 30 & 129 & 190 & 75 \\
\hline $\mathrm{P}$ & $10^{14}$ & 20 & 158 & 195 & 180 \\
\hline $\mathrm{P}$ & $10^{15}$ & 20 & 146 & 180 & 123 \\
\hline $\mathrm{P}$ & $2 \cdot 10^{16}$ & 20 & 142 & 23 & 0 \\
\hline $\mathrm{P}$ & $10^{15}$ & 30 & 145 & 192 & 190 \\
\hline As & $10^{15}$ & 20 & 134 & 353 & 240 \\
\hline As & $10^{14}$ & 30 & 160 & 120 & 380 \\
\hline As & $10^{15}$ & 30 & 137 & 114 & 191 \\
\hline As & $2 \cdot 10^{16}$ & 30 & 78 & 66 & 53 \\
\hline
\end{tabular}


(a)

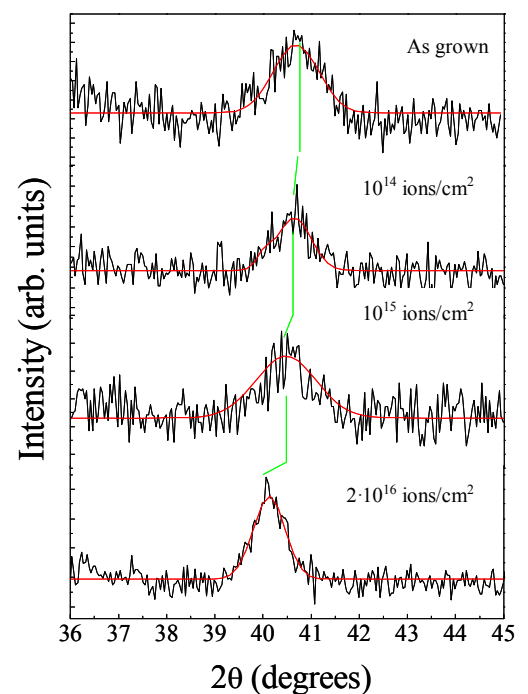

(b)

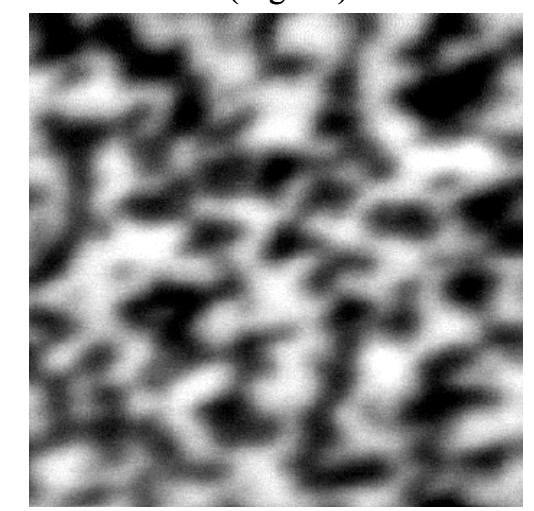

(c)

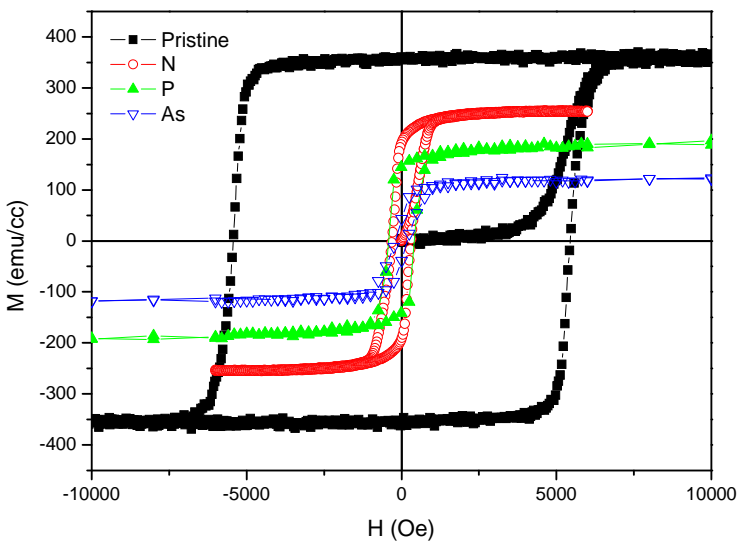

(d)

Figure 1. Structural and magnetic effects of implantation. (a) XRD scans for different CoPd multilayers implanted with nitrogen at different flux densities. (b) MFM image (lateral size: 2.9 microns) of a pristine sample. (c) SQUID magnetometic measurements of pristine sample plus samples implanted at $30 \mathrm{KeV}$ and a fluence of $10^{15}$ ions $/ \mathrm{cm}^{2}$ with different ions. The same energy and fluences is shown for comparison purposes. 

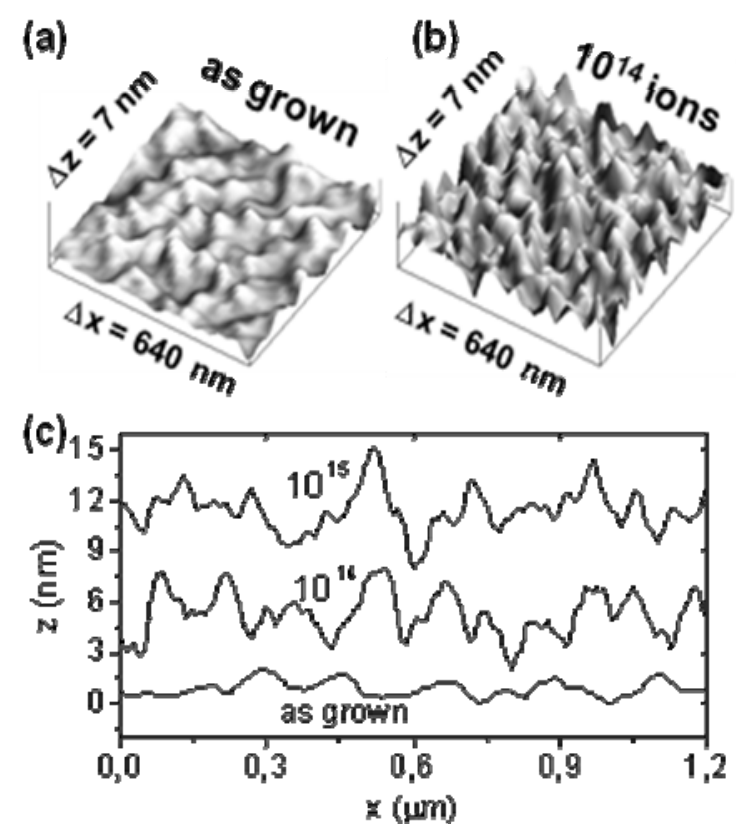

Figure 2. (a) AFM image of a pristine sample. (b) AFM image of a sample implanted with $10^{14}$ ions $/ \mathrm{cm}^{2}$ at $30 \mathrm{keV}$. (c) AFM profiles for the as grown samples and for films implanted with $10^{14}$ and $10^{15}$ ions $/ \mathrm{cm}^{2}$ at $30 \mathrm{keV}$ (vertically displaced for the sake of clarity). 
(a)

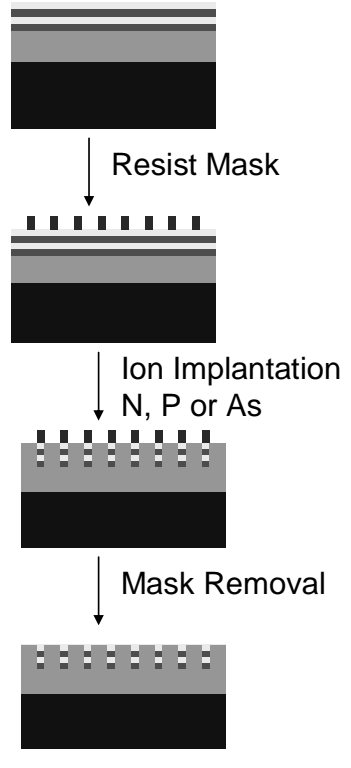

(b)

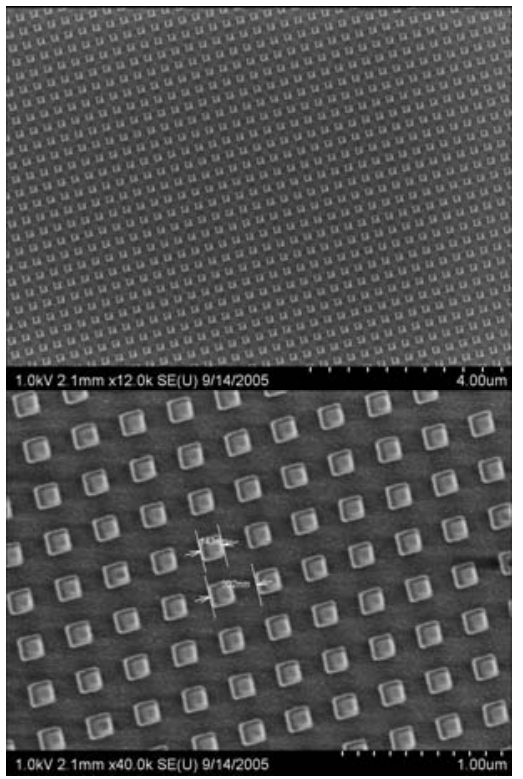

Figure 3. a) Sketch of the procedure followed in this work to modulate the magnetic anisotropy by low ion irradiation in perpendicular magnetic anisotropy multilayers b) SEM image of the photoresist mask placed on top of the films.

(a)

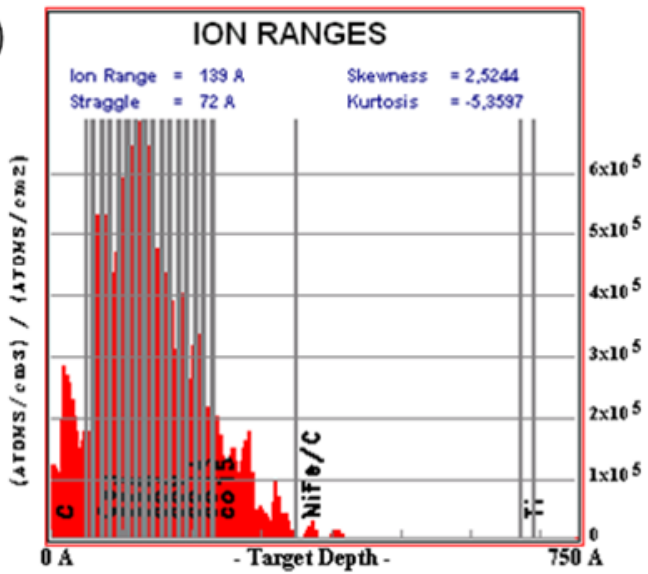

(b)

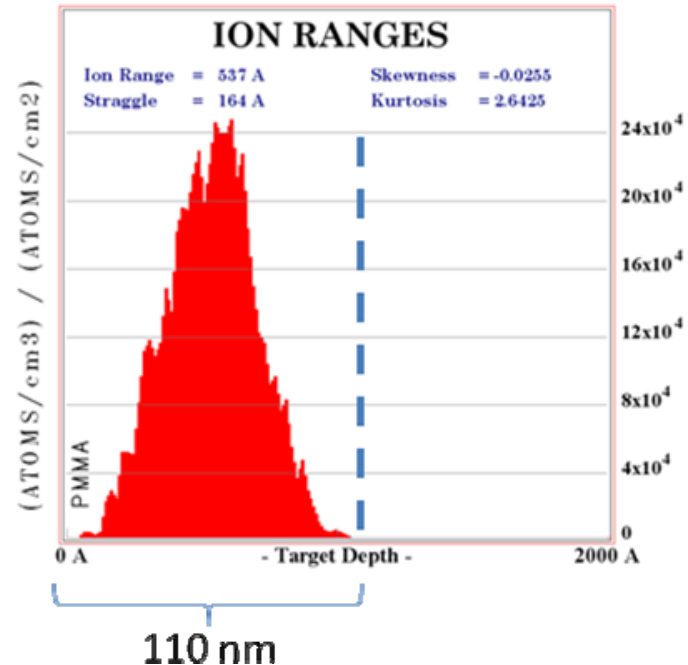

Figure 4. An example of the SRIM simulations performed in this study. a) Ion range for ions of $\mathrm{P}$ implantation at $20 \mathrm{KeV}$ in $\mathrm{Co} / \mathrm{Pd}$ multilayers. It can be observed that the $\mathrm{P}$ is distributed in a Gaussian form centered in the multilayers, b) P distribution on $200 \mathrm{~nm}$ PMMA layer at $20 \mathrm{KeV}$. As can be observed $110 \mathrm{~nm}$ is enough to stop the $\mathrm{P}$ implantation. 

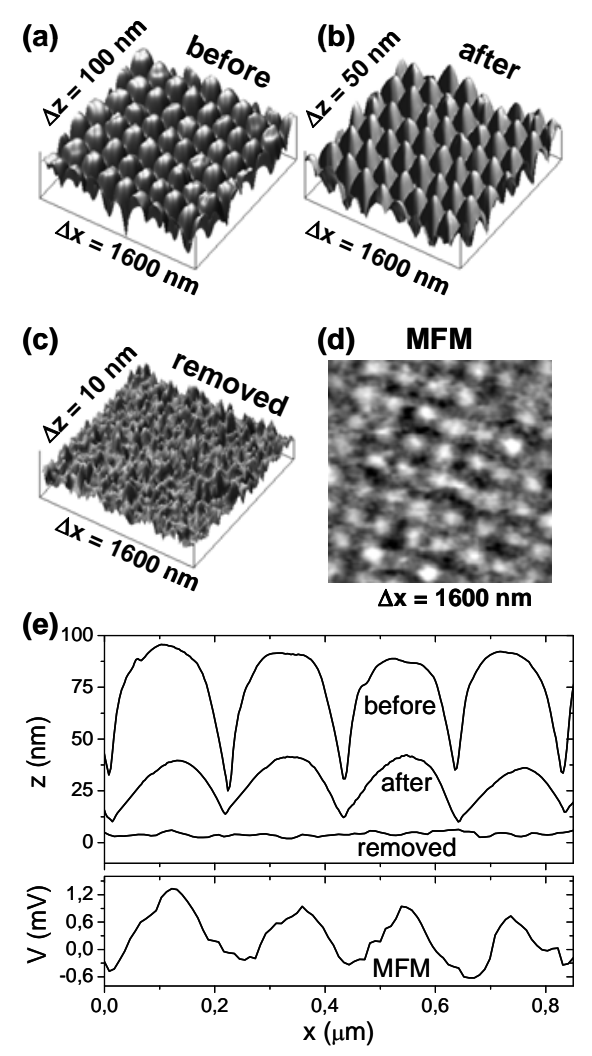

Figure 5. Magnetic patterning by implanting through a mask. (a) AFM image of the sample with the patterned resist on top before implantation. (b) AFM image of a representative sample with the patterned resist on top after $\mathrm{N}$ implantation at $30 \mathrm{KeV}$ and a fluence of $10^{15}$ ions $/ \mathrm{cm}^{2}$ (c) 3D AFM image of the sample once the patterned resist is removed. (d) MFM image showing the magnetic patterning. (e) Topographic profiles extracted from (a-c) AFM images (top) and magnetic profile extracted from (d) MFM image (down), respectively. 


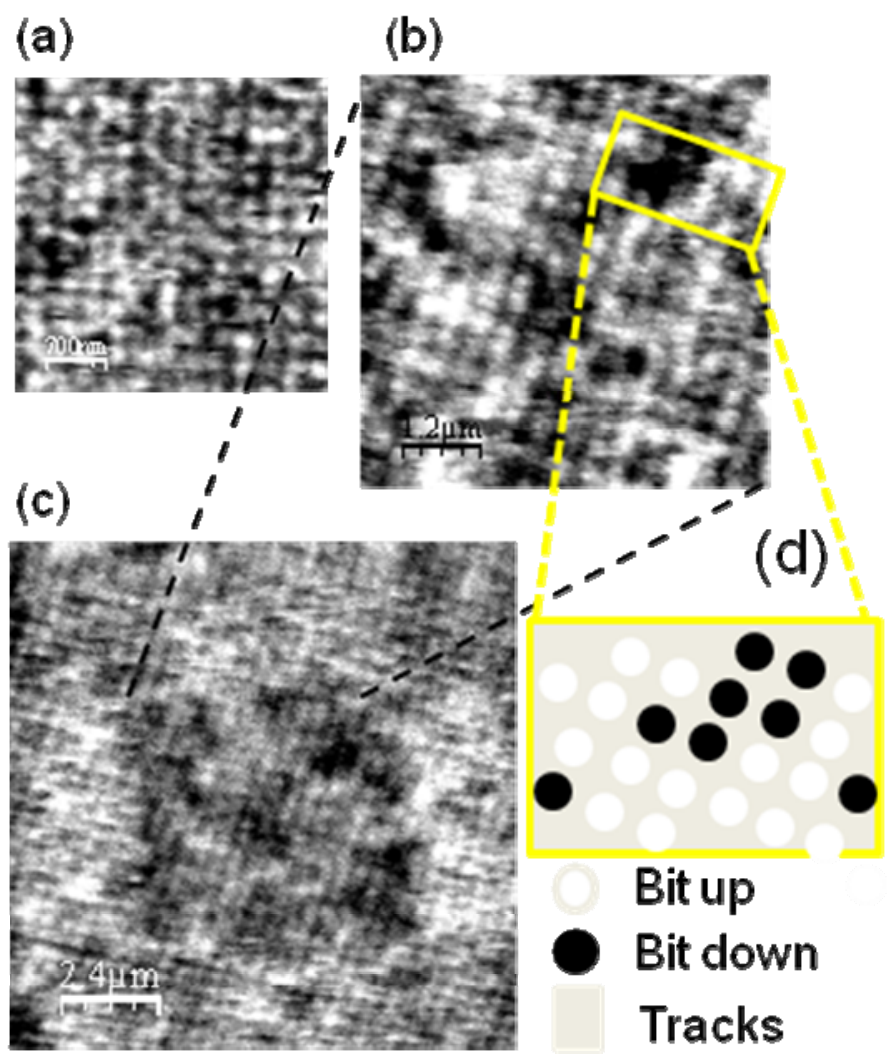

Figure 6. MFM images of the demagnetized state of samples magnetically patterned using: (a) As at $30 \mathrm{KeV}$ and $10^{15}$ ions/ $/ \mathrm{cm}^{2}$ fluence, (b) and (c) $\mathrm{P}$ at $30 \mathrm{KeV}$ and $10^{15}$ ions $/ \mathrm{cm}^{2}$ fluence, (d) sketch of how the bits looks like with respect to their orientation.

\section{REFERENCES}

\footnotetext{
${ }^{1}$ A. Moser, K. Takano, D. T Margulies, M. Albrecht, Y. Sonobe, Y. Ikeda, S. Sun, and E. E. Fullerton, J. Phys. D: Appl. Phys. 35, R157 (2002).

${ }^{2}$ E. E. Fullerton, D. T. Margulies, M. E. Schabes, M. Carey, B. Gurney, A. Moser, M. Best, G. Zeltzer, K. Rubin, and H. Rosen Appl. Phys. Lett. 77, 3806 (2000)

${ }^{3}$ E. N. Abarra, A. Inamota, H. Sato, I. Okamoto, and Y. Mizoshita Appl. Phys. Lett. 77, $2581(2000)$

${ }^{4}$ S. Iwasaki and Y. Nakamura IEEE Trans. Magn. MAG-13 1272 (1977)
} 
${ }^{5}$ G. Hu, C. T. Rettner, T. Thomson, M. Albrecht, S. Raoux, G. M. McClelland, M. W. Hart, M. E. Best, and B. D. Terris,. J. Appl. Phys, 95, 7013 (2004)

${ }^{6}$ C. A. Ross Annu. Rev. Mater. Res. 31, 203 (2001)

${ }^{7}$ S. Sun, C. B. Murray, D. Weller, L. Folks and A. Moser Science 287, 1989 (2000)

${ }^{8}$ P. Gibot, E. Tronc, C. Chanéac, J.P. Jolivet, D. Fiorani and A.M. Testa J Mag. Mag. Mat. 290-291, 555 (2005)

${ }^{9}$ BD Terris J. of Magn. and Magn. Mat. 321 512- 517 (2009)

${ }^{10}$ H. N. Bertram, H. Zhou, and R. Gustafson, IEEE Trans. Magn. 34, 1845 (1998).

${ }^{11}$ D. Weller and A. Moser, IEEE Trans. Magn. 35, 4423 (1999)

${ }^{12}$ B. D. Terris, T. Thomson, and G. Hu Microsyst Technol 13 189-196 (2007)

${ }^{13}$ C. T. Rettner, M. E. Best, and B. D. Terris, IEEE Trans. Magn. 37, 1649 (2001).

${ }^{14}$ Haast, M.A.M.; Schuurhuis, J.R.; Abelmann, L.; Lodder, J.C.; Popma, Th.J.; IEEE Trans. Magn. 34 (1998) 1006.

${ }^{15}$ G. M. McClelland, M. W. Hart, C.T. Rettner, M. E. Best, K.R. Carter, and B.D. Terris Appl. Phys. Lett. 81 (8) (2002) 1483.

${ }^{16}$ J. Moritz, B. Dieny, J. P. Nozières, S. Landis, A. Lebib and Y. Chen J. Appl. Phys. 91 (10) (2002) 7314.

${ }^{17}$ Naito, K.; Hieda, H.; Sakurai, M.; Kamata, Y.; Asakawa, K.; IEEE Trans.Magn.38 (5) (2002) 1949.

${ }^{18}$ S. Sun, C. B. Murray, D. Weller, L. Folks and A. Moser. Science 287, 1989 (2000).

19 T. Aign, P. Meyer, S. Lemerle, J. P. Jamet, J. Ferré, V. Mathet, C. Chappert, J. Gierak, C. Vieu, F. Rousseaux, H. Launois, and H. Bernas Phys. Rev. Lett. 8125 (1998), 5656. 
${ }^{20}$ Dietzel, A.; Berger, R.; Grimm, H.; Bruenger, W.H.; Dzionk, C.; Letzkus, F.; Springer, R.; Loeschner, H.; Platzgummer, E.; Stengl, G.; Bandic, Z.Z.; Terris, B.D.; IEEE Trans.Magn.38 (5) (2002) 1952.

${ }^{21}$ M. Albrecht, C. T. Rettner, M. E. Best, and B. D. Terris Appl. Phys. Lett. 83, 4363 (2003).

${ }^{22}$ E. Menendez, M. O. Liedke, J. Fassbender, T. Gemming, A. Weber, L. J. Heyderman, K. V. Rao, S. C. Deevi, S. Surinach, M. D. Baro, J. Sort, and J. Nogue's, Small, 5, 229$234(2009)$

${ }^{23}$ B.D. Belle, F. Schedin, T.V. Ashworth, P.W. Nutter, E.W. Hill, H.J. Hug, J.J. Miles, IEEE TRANSACTIONS ON MAGNETICS, 44, 3468 - 3471 (2008)

${ }^{24}$ Fassbender J, Ravelosona D and Samson A 2004 J. Phys. D: Appl. Phys. 37 R179

${ }^{25}$ C. Chappert, H. Bernas, J. Ferre, V. Kottler, J-.P. Jamet, Y. Chen E. Cambril, T, Devolder, F. Rousseaux, V. Mathet, and H. Launois, Science 280, 1919 (1998).

${ }^{26}$ J. Ferre, C. Chappert, H. Bernas, J.-P. Jamet, P. Meyer, O. Kaitasov, S. Lemerle, V. Mathet, F. Rousseaux, H. Launois J. Magn. Magn. Mater. 198-199 (1999) 191.

${ }^{27}$ D. Weller, J. E. E. Baglin, A. J. Kellock, K. A. Hannibal, M. F. Toney, G. Kusinski, S. Lang, L. Folks, M. E. Best, and B. D. Terris. J. Appl. Phys. 875768 (2000).

${ }^{28}$ C. T. Rettner, S. Anders, J. E. E. Baglin, T. Thomson, and B. D. Terris Appl. Phys. Lett. 80279 (2002)

${ }^{29}$ Stopping and Range of Ions in Matter: http://www.srim.org/

${ }^{30}$ X-ray PDF card Co 15-0806

${ }^{31}$ P. He, Z.S. Shan, J.A. Woollam, and D.J. Sellmyer, J. Appl. Phys. 73,5954 (1993).

32 A. M. Testa, D. Fiorani, M.S. Martín-González, F. Briones, J.Monserrat, H.Rorhrmann Eur. Phys. J. Appl. Phys., 38, 253-258 (2007)

${ }^{33}$ Chappert C and Bruno P J. Appl. Phys. 64, 5736 (1988) 
${ }^{34}$ Bertero G A, Sinclair R, Park C-H and Shen Z X J. Appl. Phys. 77, 3953 (1995)

${ }^{35}$ M.S. Martín-González, Y. Huttel, A. Cebollada, G. Armelles, F. Briones, Surf. Sci., $571,63(2004)$.

${ }^{36}$ I. Fernández-Martínez, M.S. Martín-González, R. González-Arrabal, R. ÁlvarezSánchez, F. Briones, J.L. Costa-Krämer J. Mag. Mag. Mat, 320, 68-75 (2008)

${ }^{37}$ J.L. Costa-Krämer, D.M. Borsa, J.M. García, M.S. Martín-González, D.O. Boerma, and F. Briones Phys. Rev. B. $\underline{69}, 144402$ (2004)

${ }^{38}$ N. Gordillo, R. Gonzalez-Arrabal, M.S. Martin-Gonzalez, J. Olivares, A. Rivera, F. Briones, F. Agulló-López and D.O. Boerma, J. Crys. Growth 310, 4362-4367 (2008).

${ }^{39}$ R. González-Arrabal, N. Gordillo, M. S. Martin-González, R. Ruiz-Bustos and F. Agulló-López J. Appl. Phys, 2010, 107, 103513

${ }^{40}$ K. Ishida and T. Nishizawa Journal of Phase Equilibria, 11, 550-560 (1990)

${ }^{41}$ M Sifkovits, H Smolinski, S Hellwig, W Weber, J. Mag. Mag. Mat. 204, 191 (1999)

${ }^{42}$ C. Peng, X. Liang, Z. Fu, S. Y. Chou, J. Vac. Sci. Technol. B 25410 (2007)

${ }^{43}$ P. Krauss, and S. Y. Chou, Appl. Phys. Lett., 71, 3175 (1997).

${ }^{44}$ Z. b. Mohamad, M. Shirai, H. Sone, S. Hosaka and M. Kodera, Nanotech. 19 (2008) 025301

${ }^{45}$ A. Thiaville, L. Belliard, D. Majer, E. Zeldov, and J. Miltat, J. Appl. Phys. 82, 3182 (1997)

${ }^{46}$ J.M. García, A. Thiaville, J. Miltat, K.J. Kirk, J.N. Chapman, F. Alouges, Appl. Phys. Lett. 79 (2001) 656

${ }^{47}$ J. Fidler, T. Schrefl, D. Suess, O. Ertl, M. Kirschner, G. Hrkac Physica B 372 (2006) $312-315$

${ }^{48}$ S. Y. Chou Proceedings of the IEEE, 85, 652-671 (1997) 
49 H.N. Bertram. Theory of Magnetic Recording, Cambridge University Press, Cambridge (1994). 